\title{
Promoting Self-determined and Informed use of Personal Health Records (PHR) among Older Adults: Assessment of Attitudes Towards the PHR and Requirements for an eLearning Platform
}

\section{Luis Perotti ( $\sim$ luis.perotti@charite.de )}

Charité - Universitätsmedizin Berlin, corporate member of Freie Universität Berlin, Humboldt-Universität zu Berlin, and Berlin Institute of Health

\section{Anika Heimann-Steinert}

Charité - Universitätsmedizin Berlin, corporate member of Freie Universität Berlin, Humboldt-Universität zu Berlin, and Berlin Institute of Health

\section{Research Article}

Keywords: Personal Health Record, eLearning, older adults, patient empowerment, eHealth

Posted Date: February 1st, 2021

DOl: https://doi.org/10.21203/rs.3.rs-150958/v1

License: (c) (1) This work is licensed under a Creative Commons Attribution 4.0 International License.

Read Full License 


\section{Abstract}

\section{Background}

Integration and implementation of Personal Health Records (PHR) in the healthcare system offers the opportunity for improved care for patients. Older adults, who often face a larger number of chronic diseases, could particularly benefit from a transparent and improved healthcare process. However, confident and self-determined use of a PHR requires a high degree of digital and content-related competence. At the same time, older adults may have little experience of using digital technology. This study aimed to assess the attitudes of older adults towards the PHR and their requirements for development of an eLearning platform designed to enable older adults to use the PHR.

Methods

To answer the research questions, semi-structured interviews with older adults (aged $\geq 65$ years) were conducted. A focus group was also set up, consisting of older adults. Sociodemographic data, previous knowledge about the PHR and willingness to use technology were additionally collected by using standardized validated questionnaires and self-developed questionnaires.

Results

While previous knowledge about the PHR was relatively low within the study population, general attitudes towards the PHR were mostly positive. The study participants mainly expressed hope for improved care, such as better communication and avoidance of duplicate examinations. At the same time, the potentially large amount of data in the system, as well as possible incomprehensibility of the content, were mentioned as concerning factors.

In terms of requirements for the eLearning platform, usability was the aspect most frequently mentioned by the participants. The subjects emphasized that the platform should consider the requirements of older adults in particular. In terms of content, help with the use of the PHR and information about its general conception, as well as access authorization, were named.

Conclusion

While the general attitude of older adults towards the PHR was relatively positive, there is a high demand for an eLearning platform enabling the target group to successfully use the PHR. Such a platform could facilitate implementation of the PHR and help older adults to actively participate in their healthcare. During development, the specific needs of the target group should be considered to ensure accessibility and usability.

\section{Background}


Personal Health Records (PHR) are intended to make processes in the healthcare system more efficient and transparent. For this purpose, data and documents are to be exchanged between service providers and the patient. The benefits of PHR use include improved cooperation, reduced duplicate examinations and improved patient care, especially for patients with chronic diseases $(1,2)$. The German PHR will be developed and released in stages, and it will store diagnoses, medical findings, medication plans, vaccination information, X-rays and other patient data. In its conception, the PHR was designed to consider the patient as the central administrator of his or her data. Use of the record is voluntary. The user decides whether a service provider may access his or her record, add content or view specific documents. Similarly, the user can independently load documents into his or her record and delete any file at will (3). These measures are intended to protect the user's privacy. At the same time, the PHR can only be used effectively if service providers can access such documents in as much detail as needed (4). As a result, adequate use of the PHR requires a considerable degree of competence on the part of end users in managing their data. On the one hand, competence or knowledge about the contents of the record is needed. Deciding which document should be accessible by which healthcare provider (and precisely when) implies that the user has an understanding of the content of each document and of how the involved parties in the healthcare system work together. Only with this knowledge is it possible to make informed and self-determined decisions. On the other hand, due to the PHR being a digital service, skills in handling digital devices and software in general are required. As a result, the advantages and disadvantages of introducing the PHR are being widely discussed. In the literature, positive effects such as the chance to promote patient empowerment and increased participation are mentioned, as well as the danger of overburdening the user (5).

With a higher prevalence of chronic diseases, corresponding increase in contact with healthcare providers and a longer history of illness, older people have the potential to accumulate a particularly large amount of data in their PHR. At the same time, introduction of the PHR may be of particular benefit to them. It offers the prospect of a better overview of their therapies, some of which are complex and involve several service providers, and improved communication. However, the data and access rights of individual service providers must also be managed by the patient, which often poses challenges for older people (6). Many older adults have limited experience of handling digital technologies, which could be a barrier to accessing and using the PHR. The training or enabling of all users of the PHR was laid down by the German legislature, and responsibility was transferred to the GKV Spitzenverband, which is the association of health insurance companies in Germany (7). The PHR has now been published (in January 2021). However, the GKV Spitzenverband has not yet provided guidelines for health insurance companies (despite there being a high demand for supplementary offers to equip users with the skills and knowledge necessary to use the PHR).

In order to address this problem, the "ePA Coach" project was founded. The project aims to develop an eLearning platform, enabling older adults to use the PHR in a self-determined and informed way. Users of the service will be able to learn the necessary skills and knowledge needed to use the PHR. Elements of microlearning, gamification and Al algorithms will be integrated in order to increase learning motivation and to make the platform as effective as possible. Studies show that gamification (especially in the 
context of social gaming) can be a suitable approach for strengthening user motivation and engagement with technology among older adults (8). Another essential part of the project is development of the eLearning platform through a participatory and iterative process, with representatives of the target group. This will ensure that the needs of the target group are precisely identified and considered in development of the service. This will raise the accessibility and effectiveness of the elearning platform. The basis for this approach is provided by studies in other countries that introduced digital health records several years ago. In these countries, research is available concerning the actual use of PHR after implementation. These studies show that overall use of digital health records is low, mainly due to the insufficient attention paid to users' needs (9). Clear communication has been identified as being particularly important, along with recognition of the benefits of PHR for older people, as this has been a key factor in poor uptake of PHR in other countries (10). Furthermore, perceived usefulness from a user's perspective is described as a driving factor for using the PHR (11).

Digital services for communication and information seeking are widely used by older adults in Germany. At the same time, use of these services for health-related purposes is less common, mostly due to a sense of mistrust about the quality of information found on the internet. Older adults tend to turn to healthcare providers to get the health information they need (12). In the context of implementation of the PHR in Germany, this fact could put an additional burden on the healthcare system, when people start seeking out doctors or pharmacists to get help in using the PHR. This will increase the need for an additional service which is independent from healthcare providers and which can help older adults acquire the skills needed for appropriate PHR use.

Against the background of this topic, it was our intention, in the study presented here, to assess what attitudes and existing knowledge older people have in the context of the Personal Health Record and what requirements might be expressed by the target group in terms of an eLearning system to help such people learn how to handle the PHR. It was also our intention to consider health-information-seeking behaviour, prior knowledge and attitudes towards the PHR. Furthermore, general use of technology by the study participants was to be recorded.

The main research questions which the presented study sought to answer are as follows:

(1) What attitudes do older adults have towards the Personal Health Record, and what experience have they had with it?

(2) What might older adults require of an eLearning system intended to help them learn how to handle the Personal Health Record?

\section{Methods}

\subsection{General}


The user-centred design process is an important instrument for target-group-oriented development of technical assistance systems (13). Accordingly, at the beginning of the project, a requirements analysis was conducted with representatives of the target group. An explorative, qualitative approach was chosen for the requirements analysis. In addition, sociodemographic data relating to the sample were collected using questionnaires.

The methodological approach was approved by the Ethics Committee of $\mathbf{X X X}$, and the study was registered in the German Register for Clinical Studies.

\subsection{Procedure and materials}

\subsubsection{Interviews}

To answer the research questions, semi-structured guideline-based interviews were conducted, as well as a focus group discussion with older adults (aged $\geq 65$ years). Collection of sociodemographic data, previous knowledge about the PHR and willingness to use technology was additionally carried out by means of a survey, using standardized validated questionnaires and self-developed questionnaires.

The goal of the qualitative interviews was to learn more about the attitudes of older people towards the PHR and to collect requirements for the eLearning system. For this purpose, older adults were recruited via the database of the Geriatrics Research Group. To be included in the study, participants had to be over 65 and had to be able to give their informed consent. Exclusion criteria were severe cognitive impairment, severe sensory impairment or having a legal representative.

After examination of the inclusion and exclusion criteria, the participants were informed, and an interview date was agreed upon, at least 24 hours after the clarifying discussion. At the beginning of the study, the subjects were contacted again, and their signed consent was obtained.

The interview guide used (developed by the study staff of the $\mathbf{X X X}$ ) contained two main topics:

1. the PHR in general (attitude, previous knowledge, advantages and disadvantages, and the need for information);

2. the eLearning system (content, presentation, device, training and support).

The interview guide included 25 main questions with up to four supplementary questions. In addition, the study included completion of three questionnaires: 1 . a self-developed questionnaire on current technology use and previous experience of the PHR; 2. a questionnaire on technology commitment (14) and 3. a questionnaire on use of mobile devices (Mobile Device Proficiency Questionnaire (15)).

The interview and answering of the questionnaires took about 1 to 1.5 hours for the participants. The interviews were recorded and then transcribed and evaluated by the Geriatric Research Group. Analysis of the transcripts was carried out after the content analysis in accordance with Mayring (16) and with the 
help of Atlas.ti 8 analysis software. The coding was done manually by two independent scientists according to the four-eye principle. In total, 29 codes were assigned.

\subsubsection{Focus group}

A focus group discussion was conducted with eight representatives of the target group. The aim of the focus group interview was to verify the previously collected requirements and to supplement these. Older people from the Senior Research Group in Berlin took part in the focus group. At the beginning, the participants were introduced to the project. Afterwards, initial results from the interviews were presented (e.g., requirements for the layout of the eLearning system) and discussed with the group. The participants' statements were recorded and evaluated. Furthermore, additional requirements were developed and included. The focus group participants filled in the same questionnaires as those who participated in the individual interviews. Two participants refused to fill in the questionnaires.

\subsubsection{Sample}

In total, 16 subjects were included in the survey. Eight were interviewed in the qualitative interviews, and eight more were part of the focus group. The subjects were between 65 and 85 years old and were well educated (mostly to a higher educational level). Thirteen subjects had already heard about the PHR prior to the study (Table 1). 


\section{Sociodemographic Data}

\begin{tabular}{|c|c|c|c|c|}
\hline & & $\begin{array}{l}\text { Interview } \\
(\mathrm{n}=8)\end{array}$ & $\begin{array}{l}\text { Focus } \\
\text { Group } \\
(\mathrm{n}=8)\end{array}$ & $\begin{array}{l}\text { Total }(\mathrm{n}= \\
16)\end{array}$ \\
\hline Age ( $\varnothing$ years, min-max) & & $\begin{array}{l}75.4(69- \\
84)\end{array}$ & $\begin{array}{l}74.1(65- \\
78)\end{array}$ & $\begin{array}{l}74.8(65- \\
84)\end{array}$ \\
\hline \multirow[t]{2}{*}{ Sex } & Male & 4 & 3 & 7 \\
\hline & Female & 4 & 5 & 9 \\
\hline \multicolumn{5}{|l|}{ Technology Usage } \\
\hline & & $\begin{array}{l}\text { Interview } \\
(\mathrm{n}=8)\end{array}$ & $\begin{array}{l}\text { Focus } \\
\text { Group } \\
(n=6)^{\star}\end{array}$ & $\begin{array}{l}\text { Total } \\
(\mathrm{n}=14)\end{array}$ \\
\hline \multirow[t]{4}{*}{ Internet usage } & Often & 8 & 6 & 14 \\
\hline & Occasionally & 0 & 0 & 0 \\
\hline & Rarely & 0 & 0 & 0 \\
\hline & Never & 0 & 0 & 0 \\
\hline \multirow[t]{4}{*}{ Computer usage } & Often & 7 & 6 & 13 \\
\hline & Occasionally & 0 & 0 & 0 \\
\hline & Rarely & 0 & 0 & 0 \\
\hline & Never & 1 & 0 & 1 \\
\hline $\begin{array}{l}\text { Technology commitment ( } \varnothing \text { score, min- } \\
\max \text { ) }\end{array}$ & & $\begin{array}{l}3.89(3.0- \\
4.4)\end{array}$ & $\begin{array}{l}4.23(4.1- \\
4.4)\end{array}$ & $\begin{array}{l}4.04(3.0- \\
4.4)\end{array}$ \\
\hline
\end{tabular}

\section{Results}

The results were divided according to the research questions into (1) the attitude of the elderly towards the PHR in general and participants' existing knowledge about the PHR; and (2) their requirements and expectations in relation to an eLearning system for teaching PHR-specific content. The results always included feedback from the individual interviews and members of the focus group.

\subsection{Expectations of the PHR}


The general attitude towards the PHR among the majority of respondents was initially positive. Respondents indicated that they would welcome its introduction and could well imagine using it. However, some respondents were sceptical about introduction of the PHR. One respondent feared that the PHR could be very confusing due to the large amount of data available. Only one respondent had a negative attitude towards introduction of the PHR. This respondent did not see any benefit in its use and could not yet "recognize its advantage" (female, 74 years).

\subsubsection{Advantages and disadvantages}

The participants were asked to name the advantages and disadvantages they associated with introduction and use of the PHR. In terms of the advantages, many factors were mentioned by the respondents. One of the most frequently mentioned advantages was improved communication, which five of the eight respondents from the individual interviews hoped might be achieved with the PHR. This included both communication between the various service providers in healthcare and doctor-patient communication.

"I also think it's good. You have a few more problems when you get older; that's just the way it is, that's all, and when everyone communicates well with each other, especially when it comes to test results, $X$-ray examinations, all those things that happen around or with people, I think it's good if you can do it electronically, if the doctor treating you has it on site and can put it all together." (female, 74 years)

The reason for this, according to the respondents, is simply the possibility of forwarding data and that all information is thus bundled centrally, in one place. In this context, three respondents assumed that central storage of data in the PHR would lead to the avoidance of duplicate examinations. One respondent saw an advantage to centralized storage in terms of the availability of all relevant data in an emergency:

"... and if i'm involved in some kind of emergency, then this can be checked there in the hospital - so I imagine that - also really fast (...), what I suffer from or which medication I must take, so this does not have to be queried, since I'm no longer able to do it." (female, 79 years)

Furthermore, most respondents assumed that time and financial resources could be saved by introduction of the PHR, in terms of physicians and the entire healthcare system $(n=6)$. Two respondents saw an advantage in the fact that the PHR itself could give them an overview of their health data and allow family members to access this information.

Three participants also expected that it would give them some peace of mind..., as patients will no longer have to rely on their memory (e.g., regarding the medication plan), which will, in turn, improve patient safety.

Furthermore, the participants were asked what disadvantages or concerns they saw in provision of a Personal Health Record and in its use. Overall, however, very few statements were made on this subject. 
"Well, if the person can influence what is written on it, then... I [would see] fewer disadvantages." (female, 69 years)

Two test persons feared that the content contained in the PHR might be incomprehensible. In this context, one respondent stated that it will involve a large amount of data, especially in the case of older people, so that the file would be very extensive. One respondent feared that with introduction of the PHR, less attention would be paid to people, since all the preliminary information relating to them would already be available. One respondent expressed concern about misuse. Two respondents stated that they currently had no concerns or saw no disadvantages.

\subsubsection{Previous knowledge}

The older adults were asked where they usually obtained information on health-related issues from. Physicians, magazines (daily newspapers, pharmacy magazines and pensioners' magazines) and the internet were mentioned as the most common source for information and were each used by three test persons. At the same time, two elderly people noted that they considered the internet to be a dubious source as it often contains contradictory information. Furthermore, the older adults informed themselves via pharmacists $(n=2)$ and books $(n=2)$.

In addition, in the subject area "Previous Knowledge", participants were asked about their previous experience with the PHR. All respondents stated that they had already heard about the PHR prior to the study. According to their statements, the respondents had heard or read about it on the internet $(n=4)$, in the press $(n=3)$ and in television reports $(n=2)$, as well as in magazines and in information material from their doctor's office ( $n=1$ in each case, with multiple answers possible).

"Not so much heard, but l've read a lot about it and have read with particular interest if it described what is being planned for it." (male, 74 years)

When asked what they already knew about the PHR, the answers were very heterogeneous. Four respondents stated that they had read that the PHR was to be introduced next year.

Two participants were able to provide information about what data would be included. Test results and prescribed medication were given as examples.

"Actually, only that it is being introduced. I didn't think it was even on the agenda for the near future. But that everyone can see all the findings, that yes, and that was actually the information I had." (female, 74 years)

In each case, one respondent had already heard about the advantages and disadvantages of the PHR and suspected that every healthcare provider would have access to the stored data (with different stakeholders possibly being given access to the stored data).

\subsubsection{Data security}


The participants were specifically asked about data protection in relation to the PHR. None of the respondents saw the topic of data protection as a worrisome issue that could hinder rollout of the PHR. The majority of participants estimated the risk of data misuse as low $(n=5)$.

"No, not really, because I think we are well-secured here in Germany. They are very careful, and sometimes that is even a hindrance, but I think that it is necessary and good." (female, 75 years)

Three participants felt that data protection was an important issue, with the security of their own data being a basic prerequisite for using the PHR. For them, the priority was ensuring that only authorized persons have access to their own data.

The respondents' low level of concern in connection with data protection issues can be attributed to various reasons. On the one hand, half of the respondents mentioned that there is always a risk of data misuse when using digital systems anyway $(n=4)$. This risk was no greater for the PHR than when using a smartphone. Two of the respondents also mentioned that they considered the probability of their data being stolen to be very low. Three of the respondents stated that they considered the public debate on this topic to be exaggerated and that it is rather obstructive for some developments. Other factors cited were that a great deal of attention is paid to data protection in Germany and that responsible authorities can be relied upon $(n=3)$. This was evident, for example, in the case of the German "Corona-Warnapp" (an application for contact tracing), which two of the participants cited as a good example of how a government-appointed app can function in a data-protection-compliant manner. The positive impression of the "Corona-Warnapp" was enhanced by the fact that problems with the system and during development were communicated transparently from the very beginning $(n=1)$.

\subsection{Requirements of an eLearning system}

Furthermore, the respondents were asked to name concrete requirements for the eLearning system, which would help them to handle the data in their PHR with confidence. The questions were about which topics they would like to be informed about, how this content should be presented, which playful or motivational elements they could envisage and what support they would need in order to use the elearning system.

\subsubsection{Learning content}

With this category, the subjects were asked which topics they would like to get information about in relation to the eLearning system. Some respondents noted that they still knew too little about the PHR to be able to respond in more detail. Six of the eight respondents expressed that they would like to know more about access rights, i.e., who has access to the data stored in the PHR from the outset and how access rights can be managed for different actors in the healthcare system (adding and removing access rights).

"That, yes; who has access, how access is enabled, and how I can decide who can see or use what." (female, 79 years) 
One respondent was concerned about whether employers would have access to the stored health data. In this context, two participants reported also wanting to be informed about who can enter data and whether data can also be entered by the users themselves. Three respondents were interested in how their own access or initial activation takes place. Two respondents firstly wanted to know, in principle, what kind of data are stored in the PHR. One respondent expressed uncertainty as to whether only the most recent health data are stored or all data "from the cradle to the grave". Two respondents were also interested in the topic area "Security Information and Protective Measures"; i.e., they wanted to know what they could do themselves to protect their data, but also what measures are already being taken to protect the stored data. One respondent named, as possible topics, whether/how data in the PHR can be deleted, whether changes will be made to the insurance card, and what the overarching goal of the PHR is.

Focus group respondents also indicated that they would like to be informed about the time period between a physician visit and the uploading of data to the PHR, whether there will be an obligation to upload certain data, who is responsible for the correctness and completeness of the uploaded data, and what the procedure is when changing health insurers. Furthermore, respondents in the focus group wanted help in assessing which data might be relevant for which physician.

\subsubsection{Layout of the eLearning system}

The respondents felt that presentation and preparation of the content covered in the eLearning system is an important factor relating to the usability of the system. The vast majority said that the content should have a clear structure (for example, in the form of diagrams etc.) $(n=6)$. In particular, content relationships and sequences should be recognizable. Seven of the eight participants preferred to have content presented as smaller blocks, with reduced information, one after the other, a format perceived as being easier to read than long passages.

"Explained as concisely as possible and not too much, yes. If what is explained is explained again, that would be too much." (male, 74 years)

Furthermore, the topic of accessibility and the low threshold of the eLearning system was discussed. In this context, simple language, easy comprehensibility of texts, images and videos, and clear, unambiguous language were each mentioned by five of the respondents and were mostly considered by them to be some of the most important points that should be taken into account during development. Likewise, the desire to avoid use of foreign words was expressed $(n=3)$. As possible functions that could increase learning success during use, it was mentioned that most content should only be explained superficially, with the possibility of accessing more in-depth information via a link $(n=5)$. Explanation of various terms (in a glossary) was mentioned here as a useful addition. One respondent also stated that the learning speed should be individually adaptable and that individual learning sections should be repeatable.

There was no clear preference among the respondents as to the form in which the content should be presented. Five respondents indicated that they would prefer text as the medium, while five respondents 
also indicated that they would consider using videos if these remained short. One respondent was completely opposed to videos, while half $(n=4)$ indicated that pictures and graphics would support the content. Three of the participants stated that they could imagine being guided through the application in the eLearning system by a virtual trainer, but that this trainer should not appear "silly" $(n=1)$.

On this topic, the focus group added that there should be an export function for the content so that it could be printed out if necessary. Furthermore, respondents in the focus group wanted to have an additional summary at the end of each chapter to increase the clarity.

Most participants stated that they would prefer a neutral, simple design for the eLearning system $(n=5)$. Too many and overly bright colours should not be used as these would be distracting $(n=4)$. Only one respondent was in favour of very strong colours, as these would increase users' interest level. However, highlighting important content using colour seemed to make sense to some $(n=3)$ and would support readability. Likewise, two participants indicated that bold text would also be appropriate for this purpose. With regard to the use of text, two respondents indicated that they considered a large font size to be important (at least 14p). Another respondent stated that an adjustable font size was necessary. Two of the participants mentioned that the text should contrast with the background. Other points mentioned were that the background should be bright $(n=1)$ and that a serious-looking font $(n=1)$ and simplifying symbols were useful $(n=1)$.

\subsubsection{Gamification elements}

Within this topic area, participants were asked about possible playful and motivational elements within the eLearning system. This involved the need for a reward system, comparison with others, and commenting on or rating training content.

Of the eight participants interviewed, seven commented on whether they would like to see integration of a reward system that would, for example, reward them by means of points for completed learning units and increase users' motivation. Five of the participants fundamentally rejected the idea that any form of reward system should be integrated into the eLearning system.

"No. There are so many points and stars already." (female, 74 years)

Two other respondents could envisage such systems having a motivating effect but, at the same time, expressed that they themselves did not need motivational elements.

"I don't need it, but I do think it motivates a lot of people." (female, 75 years)

The majority of participants $(n=6)$ rejected the idea of point systems or other rewards such as stars, while one respondent could not envisage monitoring learning success by means of a quiz. One participant pointed out that use of the eLearning system could be linked to the bonus programme of some health insurance companies, which could lead to an increase in motivation. 
Furthermore, the older adults were asked to what extent they would like to use a function in which they could rate or comment on eLearning system content. Four of the respondents indicated that they would definitely be interested in rating the content they had accessed, in order to give the creators of the texts/videos feedback on the quality and usefulness of the contributions.

"You could include that, yes - feedback on how well that succeeded in conveying [the information], e.g., if it benefited you or not. Sometimes question only requires a yes or no answer. You can find this everywhere on the internet: Did it help you when you called up something somewhere when you had a question?" (male, 84 years)

One respondent emphasized, however, that such a function would have to be voluntary. Three other participants, on the other hand, stated that they were against the introduction of a rating or comments system because they saw no need for it and would not use it.

Regarding the topic "Comparison with Other Users", the seniors were asked whether they would like to compare their learning results or learning successes within the learning program with others. Only two of the eight participants commented on this. Both rejected comparison with others.

"Yes, maybe for some this is interesting, [but] for me, less so. I just want to know if I can do it or not, yes." (female, 75 years)

\subsubsection{Support for the eLearning system}

The topic area "Operational Support" firstly asked how the older adults would like to be trained on the eLearning system once it is fully developed. Five respondents indicated that they would like to learn about the system in a group training setting.

"Personally, in a group, in a smaller group, I would like it best." (74 years, male)

Two participants specifically mentioned a group size of five to ten participants, or eight people, respectively. The advantages of group training were given as being that an exchange with like-minded people could take place, other participants' questions could be addressed, and a personal exchange could take place. In the opinion of the older adults, group training should allow for hands-on practice and trial and error. One respondent suggested visualizing the training with a presentation. Online training was not preferred by any of the respondents. Two respondents stated that an additional manual or printed material would be helpful. One respondent stated that a manual would be sufficient. This respondent saw a manual as having the advantage of enabling individuals to easily and quickly pass on information to relatives or friends. One respondent declined printed material or would print out the information himself/herself if needed.

On this topic, the respondents were then asked what form of help functions there should be within the eLearning system. 
The majority of respondents stated that they would be happy to resolve technical or content-related difficulties over the phone $(n=6)$. This kind of problem-solving was perceived as being easier because questions and comments can be addressed directly.

"Yeah, so maybe if it seems a little weird or not right, or I can't do it, I would want to maybe have a phone number where I could then call, and together, both sides could resolve it." (female, 74 years)

Likewise, six participants could imagine making use of a "frequently asked questions" (FAQ) service. However, such an FAQ function should be reduced, i.e., not containing too much information. At the same time, one participant stated that it was her experience that FAQs often did not provide the exact question or answer she was looking for. Email was mentioned by five respondents as being another way of contacting a support service. This was described as being helpful because it could enable a quick response to be obtained for information needed with regard to one's own problem, and it could also be used at weekends and at night.

Two participants expressed the desire to have a manual explaining correct use of the elearning system. Other possible contact points for help with problems were named as being one's own family $(n=1)$, a chat function $(n=1)$ and a forum for questions $(n=1)$.

Respondents in the focus group additionally mentioned the desire to have a specific person to contact for concrete content-related problems.

\section{Discussion}

In the present study, the attitudes of older adults towards implementation and use of the PHR were examined, as well as the requirements for an eLearning platform enabling older adults to use the PHR. Sixteen older adults were interviewed in total. All participants reported that they were interested in the PHR and had been looking for information about it and its implementation in Germany prior to this study. At the same time, little knowledge about it was evident among the participants, who reported difficulties in gathering adequate information about the German version of the PHR. Although the study by Paccoud et al. found that individuals aged 65 or older are associated with a lower desire to use the PHR, this was not found to be the case in the population of the study presented here (17). All the participants in this study reported wanting to use the PHR after its release. These findings strengthen the fact that there is a need for a platform which can provide bundled information about the PHR to the target population of older adults. When considering experiences of PHR implementation in other countries, it becomes clear that a lack of accessible information could threaten successful implementation of the PHR with the target group of older adults. Price et al. and Ose et al. identified clear communication about the usefulness of the PHR (9) and attention to users' needs (10) as key factors in this regard in their research. Measures to inform older adults about usage and PHR content is therefore crucial to avoid non-use of this service which is designed to enable users to actively participate in their own healthcare. Non-use of the PHR could also be a waste of health-system resources and could create an additional burden for healthcare providers if they cannot rely on a specific PHR to be complete and error-free (18). 
The participants in this study also expressed a considerable number of perceived advantages to proper use of the PHR. Although this may not be true for the whole target population, it hints at the possibility that older adults see a large potential benefit in implementation of the PHR. This may be important because the general attitude of older adults towards the PHR prior to its implementation might be an important factor, which exerts a large effect on actual usage behaviour. As studies like the research by Hussein show, there is a high correlation between attitude and intention to use in the context of new digital systems and technology in mHealth (19). A lack of helpful information and resulting frustration and confusion in the context of the PHR may compromise the positive attitude of older adults who actually see the PHR as an opportunity for improved healthcare. This problem is particularly important for a system like the PHR, which considers users to be the central actors and relies on them to actively participate in its use. Due to the PHR having been released in 2021 without a broad programme in place to provide older adults with key information, this survey of user attitudes and experiences strengthens the identified need for a designated learning solution.

This realization and the statements of the study participants put a special emphasis on mediation of the PHR's usefulness to older adults. However, appropriate use of the PHR not only depends on the knowledge and attitudes of users but also the existence of competences, which are a prerequisite for use. For the PHR, required competences could mainly be identified on two levels: use of the underlying technology, and management and understanding of the content.

Most of the older adults in this study reported using technology very frequently and did not report any mayor difficulties using digital devices or software. Yet this cannot be considered as a representative fact for the whole population of older adults in Germany as there are many different levels of technology usage present. The target population is very diverse, and technology usage differs within different age groups and places of residence, with a large difference existing between rural and urban areas (20). Study participants did not report a need for the emerging eLearning platform to teach basic technology usage skills. Still, mediation of usage skills may be integrated in the coaching system in the future, making it possible to reach older adults who have little to no previous experience of using digital services and enabling them to use the PHR as well.

In terms of PHR content, the study participants identified various skills and competences which they would like the eLearning platform to address. It became clear that the participants had very diverse knowledge about medical procedures, the basic functionality of the PHR and the data to be stored in it. The older adults reported a high demand for these kinds of skills and knowledge to be taught within the eLearning system. This was therefore identified as a key component of the coaching system.

The "ePA Coach" eLearning system should consider the needs of older adults and adopt their preferences in as many aspects as possible. The key factor identified by the study participants when asked about their wishes for and expectations of the eLearning program was usability. Information within the system should be presented in an easy-to-understand way for older adults and should be clearly structured. Lowthreshold information transfer for older adults could be identified: short sentences; no use of foreign 
words; reduced information; and small, repeatable learning segments (a microlearning approach) etc. Similar aspects have been identified in other literature (21). The design and presentation preferences expressed by the target group in relation to the platform were also similar to those found in the literature (22): a strong colour contrast between text and background; a large, adaptable font size; and a high level of clarity etc.

In general, the study participants reported that they wanted the eLearning system to look and feel plain, practical and neutral, and thereby be serious and reliable. At the same time, most of the older adults spoke out against the integration of advertisements and gamification elements in the eLearning platform. Although gamification elements are specifically described as being an appropriate and effective way to raise motivation in older adults (8), the interviewees in this survey were sceptical about incorporation of gamification elements. A possible explanation could be that the eLearning program is designed to inform users about health-related issues involving sensitive data and is related to an application developed by the state. These factors were reported as being the main factors in respect of which the platform had to demonstrate complete trustworthiness, so generally valid preferences (among older adults) for learning systems and software in general may not be applicable in this context, where the reliability of the content is a top priority.

Although many of the study participants had sought information about health-related issues online, they reported having experienced difficulty identifying reliable sources on the internet. They expressed the feeling that much of the content that can be found online does not seem trustworthy and needs to be scrutinized. This previous experience with online information in the context of health issues may have led to scepticism and influenced the participants' reported requirement for the learning platform to appear as serious and trustworthy as possible.

Against this background, it was surprising that none of the study participants had major concerns about the safety of their data within the PHR. Although there was a clear demand for eLearning to be informative about potential data-security measures and issues, the older adults expressed a high level of trust in the PHR developers' ability to create a safe system. While data security is frequently discussed in the public domain, it does not seem to be a particular concern for older adults. This finding contrasts with other literature such as the study conducted by Ware et al., in which older adults expressed concerns about data security associated with eHealth technologies (23).

\section{Limitations}

Although a number of measures were taken during design of the study to avoid potential bias, there are some limitations to be considered when interpreting the presented results. Overall, the study participants were well educated and had an affinity for technology. The results are therefore not valid for the target group in general. Furthermore, the study population was rather small and not very diverse in terms of education level and interest in the PHR. The study results should therefore be interpreted with these limitations in mind. 


\section{Conclusion}

The survey of older adults showed that there is a high demand for an eLearning program that conveys important information and teaches skills as well as competences in connection with the PHR and its use for older adults. This platform could play an important role in successful implementation of the PHR in the healthcare of older adults. In development of the eLearning platform, the specific needs of the target group should be considered to ensure accessibility and usability. In the first step, the eLearning system should focus on mediation of important information and skills needed in order to use the PHR. In the future, the platform could also consider training older adults in general use of technological devices and digital software.

\section{List Of Abbrevations}

PHR Personal Health Record

\section{Declarations}

\section{Funding}

This study was funded under the ePA Coach project by the Federal Ministry of Education and Research, Germany (16SV8483).

\section{Conflict of interest}

The authors declare that they have no financial or personal relationships that may influence (bias) their work and no conflict of interest.

\section{Compliance with ethical standards}

Ethical approval to conduct the study was obtained from the Charité - Universitätsmedizin Berlin ethical review board (EA1/142/20). All procedures performed involving human participants were undertaken in accordance with the ethical standards of the relevant institutional and/or national research committee and in accordance with the 1964 Declaration of Helsinki and its later amendments or comparable ethical standards. Informed consent was obtained from all individual participants included in the study.

\section{Authors' contributions}

Both authors conceptualized and designed the study. Both authors contributed to data collection. Both authors conducted and interpreted the interviews as well as the statistical analyses. Both authors 
contributed to the writing of the draft article. Both authors revised the intellectual content of the article. Both authors read and approved the final manuscript.

\section{Availability of data and materials}

Data relating to the interviews and questionnaires supporting the conclusions of this article are available from the corresponding author upon reasonable request.

\section{References}

1. Hong MK, Wilcox L, Feustel C, Wasileski-Masker K, Olson TA, Simoneaux SF. Adolescent and Caregiver Use of a Tethered Personal Health Record System. 2017;11.

2. Robotham $D$. Electronic personal health records for people with severe mental illness; a feasibility study. 2015;8.

3. Fragen und Antworten zur elektronischen Patientenakte [Internet]. Bundesgesundheitsministerium. [Accessed: 14 Oct. 2020]. Available from:

https://www.bundesgesundheitsministerium.de/elektronische-patientenakte.html

4. Weis A, Pohlmann S, Poss-Doering R, Strauss B, Ullrich C, Hofmann H, et al. Caregivers' role in using a personal electronic health record: a qualitative study of cancer patients and caregivers in Germany. BMC Med Inform Decis Mak. Dec. 2020; 20(1): 158.

5. Crameri K-A, Maher L, Van Dam P, Prior S. Personal electronic healthcare records: What influences consumers to engage with their clinical data online? A literature review. Health Inf Manag J. 10 Jan 2020;183335831989536.

6. Pushpangadan S, Seckman C. Consumer perspective on personal health records: A review of the literature. Online J Nurs Inform. 1 Jan. 2015; 19.

7. § 20k SGB V Förderung der digitalen Gesundheitskompetenz [Internet]. [Accessed: 14 Nov. 2020]. Available from: https://www.sozialgesetzbuch-sgb.de/sgbv/20k.html

8. Koivisto J, Malik A. Gamification for Older Adults: A Systematic Literature Review. The Gerontologist. 2020;13.

9. Ose D, Kunz A, Pohlmann S, Hofmann H, Qreini M, Krisam J, et al. A Personal Electronic Health Record: Study Protocol of a Feasibility Study on Implementation in a Real-World Health Care Setting. JMIR Res. Protoc. 2017;11.

10. Price MM, Pak R. Older adults' perceptions of usefulness of personal health records. 2012;14.

11. Niazkhani Z, Toni E, Cheshmekaboodi M, Georgiou A, Pirnejad H. Barriers to patient, provider, and caregiver adoption and use of electronic personal health records in chronic care: a systematic review. BMC Med. Inform. Decis. Mak. Dec. 2020; 20(1): 153.

12. Chaudhuri MS, Demiris DG. Examining Health Information-Seeking Behaviors of Older Adults. 2014; 14. 
13. Shluzas LA, Steinert M, Katila R. User-Centered Innovation for the Design and Development of Complex Products and Systems. In: Leifer L, Plattner H, Meinel C, editors. Design Thinking Research: Building Innovation Eco-Systems [Internet]. Cham: Springer International Publishing. 2014; pp. 13549. Available from: https://doi.org/10.1007/978-3-319-01303-9_10

14. Neyer FJ, Felber J, Gebhardt C. Entwicklung und Validierung einer Kurzskala zur Erfassung von Technikbereitschaft. : 14.

15. Roque NA, Boot WR. A New Tool for Assessing Mobile Device Proficiency in Older Adults: The Mobile Device Proficiency Questionnaire. J Appl Gerontol. 2016 Apr. 11;37(2):131-56.

16. Mayring P. Qualitative Inhaltsanalyse. In: Mey G, Mruck K, editors. Handbuch Qualitative Forschung in der Psychologie [Internet]. Wiesbaden: VS Verlag für Sozialwissenschaften. 2010; pp. 601-13. Available from: https://doi.org/10.1007/978-3-531-92052-8_42

17. Paccoud I, Baumann M, Le Bihan E, Pétré $B$, Breinbauer $M, B$ Böhme $P$, et al. Socioeconomic and behavioural factors associated with access to and use of personal Health Records. BMC Med. Inform. Decis. Mak. 13 Jan. 2021; 21(1): 18.

18. Greenhalgh T, Hinder S, Stramer K, Bratan T, Russell J. Adoption, non-adoption, and abandonment of a personal electronic health record: case study of HealthSpace. BMJ. 16 Nov. 2010; 341(nov16 1): c5814-c5814.

19. Hussein Z. Consumer Attitude: Does It Influence the Intention to Use mHealth? Procedia Comput. Sci. 2017; 5 .

20. Greenberg AJ, Haney D, Blake KD, Moser RP, Hesse BW. Differences in Access to and Use of Electronic Personal Health Information Between Rural and Urban Residents in the United States: ePHI Use in Rural and Urban Patients. J. Rural Health. Feb. 2018; 34:s30-8.

21. Kuerbis A, Mulliken A, Muench F, A. Moore A, Gardner D. Older adults and mobile technology: Factors that enhance and inhibit utilization in the context of behavioral health. Ment Health Addict Res [Internet]. 2017 [Accessed: 14 Jan. 2021]; 2(2). Available from: http://oatext.com/Older-adults-andmobile-technology-Factors-that-enhance-and-inhibit-utilization-in-the-context-of-behavioralhealth.php

22. Mannheim I, Schwartz E, Xi W, Buttigieg SC, McDonnell-Naughton M, Wouters EJM, et al. Inclusion of Older Adults in the Research and Design of Digital Technology. Int. J. Environ Res Public Health. 2 Oct. 2019; 16(19): 3718.

23. Ware P, Bartlett SJ, Paré G, Symeonidis I, Tannenbaum C, Bartlett G, et al. Using eHealth Technologies: Interests, Preferences, and Concerns of Older Adults. Interact J. Med. Res. 23 Mar. 2017; 6(1): e3. 